\title{
Prolyl hydroxylase 3 controls the intestine goblet cell generation through stabilizing ATOH1
}

\author{
Yi-ming $\mathrm{Xu}^{1} \cdot$ Qiang Gao ${ }^{1} \cdot$ Jin-zhao Zhang ${ }^{1} \cdot$ Yun-tao Lu${ }^{1} \cdot$ Dong-ming Xing ${ }^{2,3} \cdot$ Yan-qing Qin ${ }^{1} \cdot J^{\text {Jing Fang }}{ }^{2,3}$
}

Received: 31 May 2019 / Revised: 2 January 2020 / Accepted: 7 January 2020 / Published online: 20 January 2020

(c) The Author(s), under exclusive licence to ADMC Associazione Differenziamento e Morte Cellulare 2020

\begin{abstract}
Intestinal epithelia self-renew constantly and generate differentiated cells such as secretary goblet cells. The intestine goblet cells secrete gel-forming mucins that form mucus to create a barrier of defense. We reported previously that loss of prolyl hydroxylase (PHD) 3 led to disruption of the intestinal epithelial barrier function. However, the underlying mechanism remains elusive. Here, we demonstrate that PHD3 controls the generation of intestine goblet cell. We found that genetic ablation of Phd3 in mice intestine epithelial cells reduced the amount of goblet cells. Mechanistically, PHD3 bounds the E3 ubiquitin ligase HUWE1 and prevented HUWE1 from mediating ubiquitination and degradation of ATOH1, an essential driver for goblet cell differentiation. The prolyl hydroxylase activity-deficient variant PHD3(H196A) also prevented ATOH1 destruction. A genetic intestine epithelial PHD3(H196A)-knockin had no effect on ATOH1 expression or goblet cell amount in mice, suggesting that the PHD3 prolyl hydroxylase activity is dispensable for its ability to control ATOH1 expression and goblet cell generation. In dextran sulfate sodium (DSS)-induced experimental colitis, PHD3-knockout rather than PHD3 (H196A)-knockin sensitized the mice to DSS treatment. Our results reveal an additional critical mechanism underlying the regulation of ATOH1 expression and goblet cell generation and highlight that PHD3 plays a role in controlling intestine goblet cell generation in a hydroxylase-independent manner.
\end{abstract}

\section{Introduction}

Prolyl hydroxylase domain proteins (PHDs) are dioxygenases that use oxygen and 2-oxoglutarate as co-substrates. PHDs are involved in cellular response to oxygen by hydroxylating conserved prolyl residues of hypoxia-inducible factor (HIF) $\alpha$,

These authors contributed equally: Yi-ming Xu, Qiang Gao

Edited by R. Johnstone

Supplementary information The online version of this article (https:// doi.org/10.1038/s41418-020-0490-7) contains supplementary material, which is available to authorized users.

Jing Fang

jfang2018@163.com

1 Shanghai Institute for Nutrition and Health, Chinese Academy of Sciences, University of Chinese Academy of Sciences, Shanghai 200031, China

2 Cancer Institute, the Affiliated Hospital of Qingdao University, Qingdao 266061, China

3 Cancer Institute, Qingdao University, Qingdao 266061, China leading to HIF $\alpha$ destruction [1-3]. Under hypoxia, the enzymatic activity of PHDs is inhibited, resulting in accumulation of HIF $\alpha$. The PHD family consists of PHD1 (EglN2), PHD2 (EglN1), and PHD3 (EglN3). Each isoform displays its own tissue and cell line-specific expression pattern, as well as its own particular subcellular distribution [4]. In addition to HIF $\alpha$, PHDs have other targets and may function in a prolyl hydroxylase-independent manner [5-14].

The intestinal epithelium is a single-cell layer that mediates the functions not only in absorption of nutrients but also in maintenance of barrier function. It is highly organized and constantly renewed by intestinal stem cells located in crypts. The intestinal stem cells give rise to a pool of transitamplifying cells. The transit-amplifying cells are highly proliferative and have a tendency to differentiate into a specific type of cell such as absorptive enterocytes and secretary cells including goblet, enteroendocrine, and Paneth cells. The intestinal goblet cells are simple columnar epithelial cells and their major function is to secrete gel-forming mucins that form mucus. The mucus layer overlies the intestinal epithelium and acts as a barrier of defense against physical and chemical injury, maintaining intestinal homeostasis [15]. Notch signaling plays a critical role in controlling differentiation of 
A

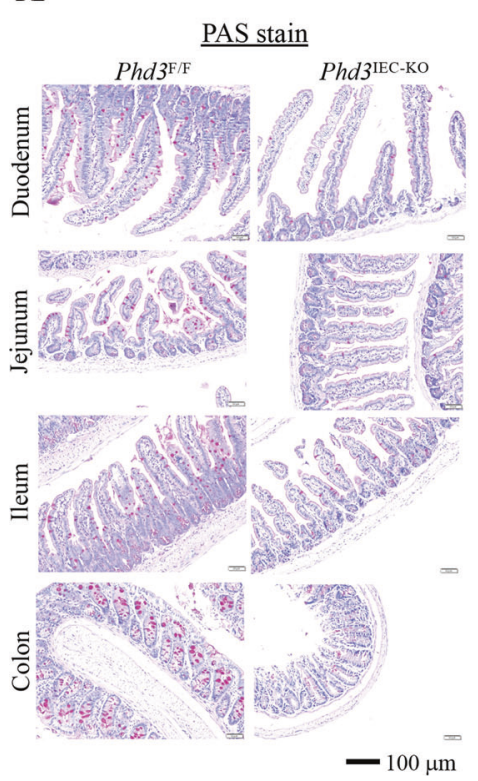

B

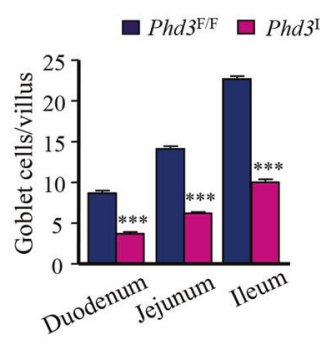

C

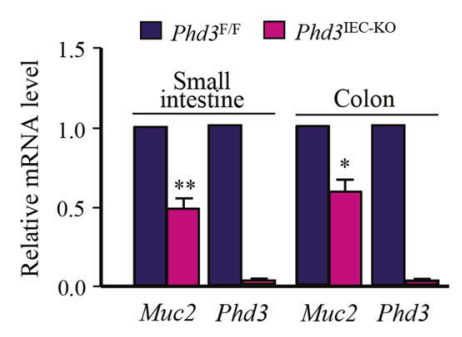

D

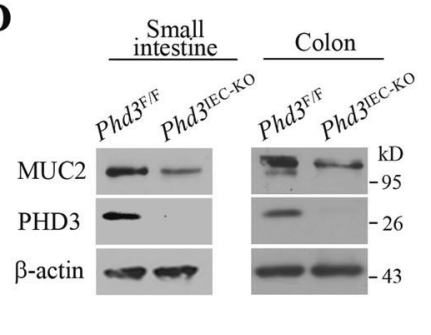

$\mathbf{E}$

$\underline{\text { MUC2 stain }}$

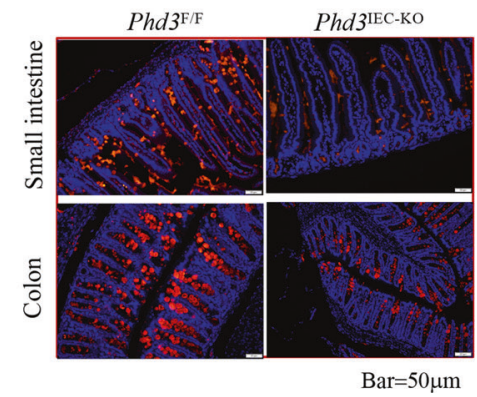

Fig. 1 PHD3 deficiency reduced goblet cell number in mice intestines. a PAS stain of epithelia of mice duodenum, jejunum, ileum, and colon. Male mice at age of 5 weeks old (the body weight of the mice is within normal limits) were selected randomly $(n=6)$. b Goblet cell number of epithelia of mice duodenum, jejunum, ileum, and colon $(n=6)$. c Determination of mRNA level of Muc2 $(n=6)$. d Determination of Muc2 in mice intestinal epithelia cells by western blot by using Muc2(ab134119) antibody. e Immunofluorescent stain of mice intestinal epithelia with Muc2(H300) antibody. ${ }^{*} P<0.05$; $* * P<0.01$; $* * * P<0.001$. intestinal epithelial cells $[16,17]$. It inhibits differentiation of secretory goblet, Paneth, and enteroendocrine cells, and induces that of absorptive enterocytes [18]. The transcription factor hairy and enhancer of split-1 (HES1) whose transcription is activated by notch intracellular domain (NICD; activated Notch-1) [19], induces enterocytes differentiation and inhibits secretory cell generation [20]. The transcription factor atonal homolog 1 (ATOH1, also known as MATH1 and HATH1) plays a critical role in secretory cell differentiation [18, 21, 22]. It is thought that Notch-induced HES1 acts as a transcriptional repressor to silence ATOH1 transcription and suppress secretory cell fate [23].

A few studies indicate that PHDs are involved in regulation of intestinal barrier function in mice [24, 25]. We found that genetic ablation of PHD3 in mice intestinal epithelial cells impaired the epithelial barrier function, leading to bowel inflammation [26]. Analysis of clinical samples indicated that downregulation of PHD3 was associated with progression of human ulcerative colitis [26]. These results suggest that PHD3 plays a role in maintaining intestinal homeostasis. Though PHD3 is found to be linked to maintenance of intestinal homeostasis, the underlying mechanism remains to be elucidated. In this paper, we demonstrate that PHD3 plays a role in regulation of intestinal goblet cell generation. We found that genetic ablation of PHD3 reduced the amount of mice intestinal goblet cells. Mechanistic studies indicate that PHD3 controls goblet cell generation through stabilizing ATOH1. Our results reveal a new mechanism underlying the regulation of goblet cell generation through PHD3.

\section{Results}

\section{Loss of PHD3 reduced the number of goblet cells in mice intestine}

As the intestinal goblet cells play an important role in protection of intestinal epithelium, we asked whether PHD3 influenced intestinal barrier function through goblet cells. To know this, we determined goblet cells generation by employing intestinal epithelial PHD3-knockout ( $\left.P h d 3^{\mathrm{IEC}-\mathrm{KO}}\right)$ mice. Male mice at age of 5 weeks were examined. Using periodic Acid-Schiff (PAS) staining to detect mucosubstances that are normally restricted to goblet cells, we found that the number of PAS-positive cells decreased significantly in both the small intestine and colon epithelium of $P h d 3^{\mathrm{IEC}-}$ KO mice as compared with control littermates (Fig. 1a, b). Mucin 2 (Muc2), a member of the mucin protein family, is particularly prominent in the gut where it is secreted from goblet cells [22]. Loss of PHD3 caused a decrease of Muc2 mRNA in mice intestine epithelial cells (Fig. 1c). Immunoblotting results show that Muc2 protein was also decreased in intestine epithelial cells of $P h d 3^{\mathrm{IEC}-\mathrm{KO}}$ mice (Fig. 1d). We did immunostaining of mice intestine tissues with Muc2 antibody and found that the Muc2-possitive cells 
A

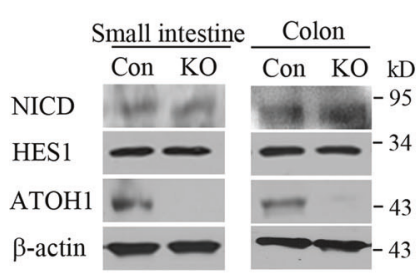

B
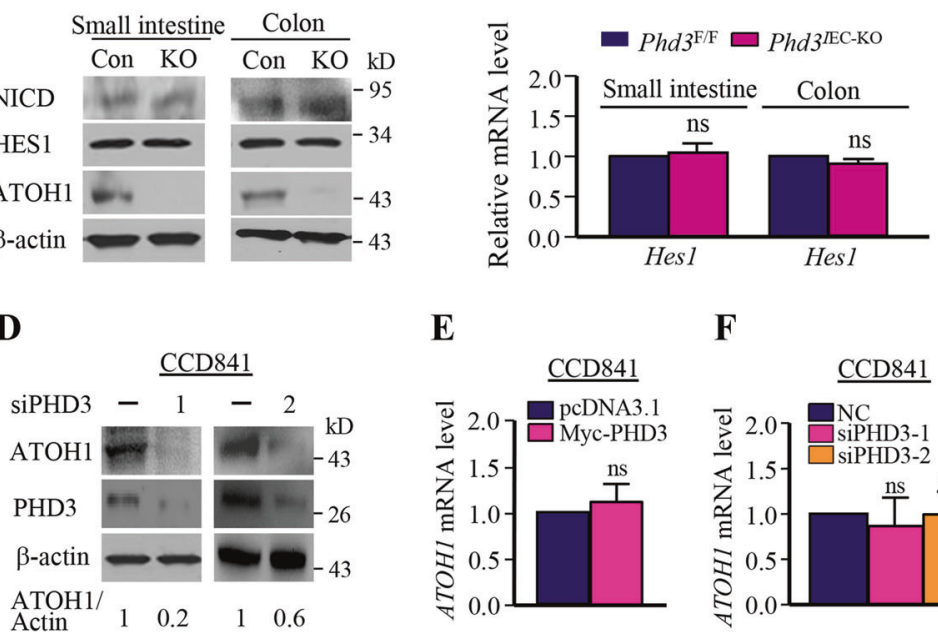

$\mathbf{E}$

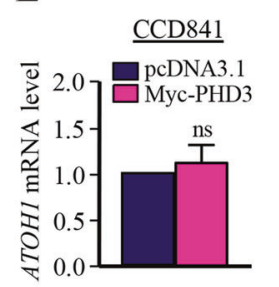

$\mathbf{F}$

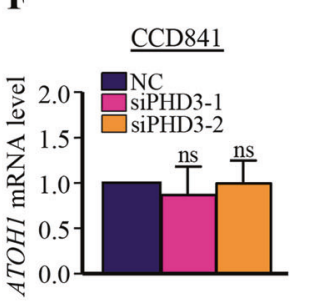

C

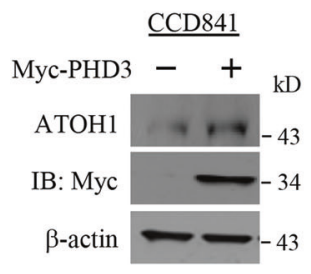

G

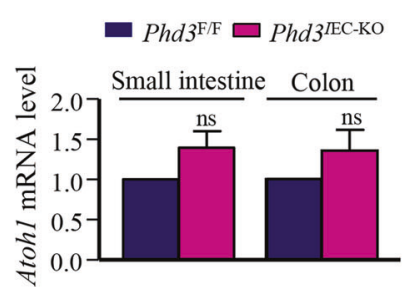

$\mathbf{J}$

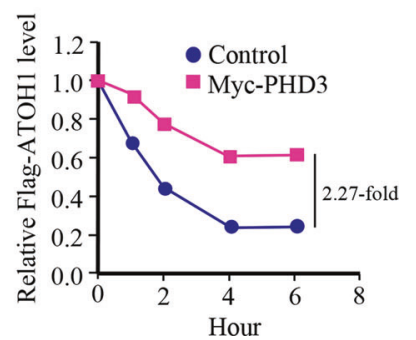

Fig. 2 PHD3 stabilized ATOH1. a Determination of NICD, HES1, and ATOH1 in intestinal epithelial cells by western blot. The small intestinal epithelial and colonic epithelial cells were isolated as described in "Methods". b Determination of the mRNA levels of Hes 1 in intestinal epithelial cells $(n=6)$. c CCD841 cells were transfected with myc-PHD3 vector. After $24 \mathrm{~h}$, the cells were harvested for determination of ATOH1 by western blot. $\mathbf{d}$ The cells were transfected with PHD3 siRNA oligos as indicated. After $48 \mathrm{~h}$, the cells were harvested for determination of ATOH1 by western blot. e CCD841 cells were transfected with myc-PHD3 vector. After $24 \mathrm{~h}$, the cells were harvested for determining $A T O H 1$ expression by qPCP. f CCD841 cells were transfected with PHD3 siRNA oligos. After $48 \mathrm{~h}$,

were decreased in intestinal epithelia of $P h d 3^{\mathrm{IEC}-\mathrm{KO}}$ mice (Fig. 1e). Together, these results suggest that PHD3 is critical for the generation of intestinal goblet cells.

\section{Loss of PHD3 decreased ATOH1 in intestinal epithelial cells}

We determined the possible mechanism underlying the regulation of goblet cell generation by PHD3. As the Notch signaling plays an important role in goblet cell generation $[16,17]$, we determined the protein level of NICD in intestine epithelial cells from $P h d 3^{\mathrm{IEC}-\mathrm{KO}}$ mice. The results show that loss of PHD3 had little effect on the protein level of NICD (Fig. 2a). In consistence with the results, PHD3-loss did not influence the protein level of HES1 (Fig. 2a). Loss of PHD3 had no effect on mRNA level of Hesl, either (Fig. 2b). Thus, the cells were harvested for determining ATOH1 expression by qPCR. g Determination of mRNA levels of Atohl in mice intestinal epithelial cells $(n=6)$. h 293 T cells were transfected with Flag-ATOH1 or FlagATOH1 plus myc-PHD3 vector. After $24 \mathrm{~h}$, the cells were harvested for western blot. i $293 \mathrm{~T}$ cells were transfected with Flag-ATOH1 or Flag-ATOH1 plus myc-PHD3 plasmid. After $24 \mathrm{~h}$, cycloheximide $(\mathrm{CHX})(10 \mu \mathrm{g} / \mathrm{ml})$ was added and the cells were incubated for different time interval as indicated. $\mathbf{j}$ The relative Flag-ATOH1 level was determined by measuring the density of Flag-ATOH1 band and normalized to that of actin. The relative Flag-ATOH1 protein level at the starting time is designated as 1 .

PHD3 may not regulate goblet cell formation through NICD/ HES1 axis. Interestingly, we found that knockout of PHD3 resulted in a decrease of ATOH1 protein (Fig. 2a). As ATOH1 is the key factor that controls differentiation of intestinal goblet cells, our results suggest that PHD3 regulates goblet cell generation through ATOH1. It is known that ATOH1 is also required for differentiation secretory cell [18, 21, 22], including Paneth and enteroendocrine cells [22, 27, 28]. So, we determined these cells. The results show that PHD3 knockout reduced the amount of Paneth and enteroendocrine cells in small intestine (Supplementary Fig. 1).

\section{PHD3 stabilized ATOH1}

To understand the regulation of ATOH1 expression by PHD3, we determined the effect of PHD3 on expression of 


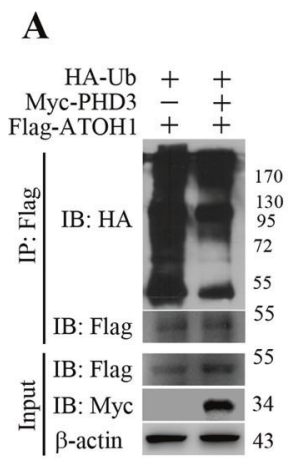

D

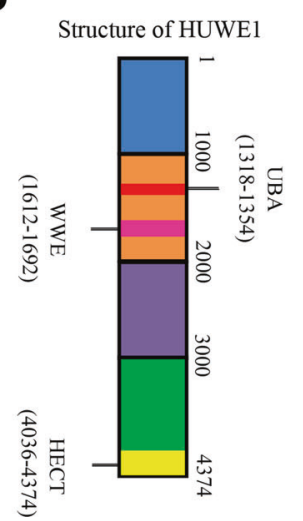

$\mathbf{E}$

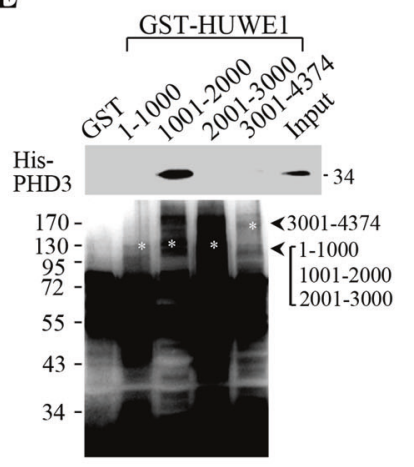

Fig. 3 PHD3 bound HUWE1. a Overexpression of PHD3 reduced ATOH1 ubiquitination. 293T cells were transfected with Myc-PHD3 or Myc-PHD3 plus Flag-ATOH1 vectors in the presence of HA-Ub vector. After $24 \mathrm{~h}$, the cells were treated with MG132 $(10 \mu \mathrm{M})$ for $4 \mathrm{~h}$. Cell lysates were prepared for immunoprecipitation. b 293T and CCD841 cells were transfected with control or Myc-PHD3 vectors. After $24 \mathrm{~h}$, the cells were harvested for determining HUWE1 by western blot. c PHD3 bound HUWE1. Proteins from CCD841 cells were used for the co-immunoprecipitation assay. $\mathbf{d}$ The schematic of construction of truncated HUWE1. e His-PHD3 bound GST-HUWE1 (1001-2000). GST-pull-down experiment was done as described in the
F

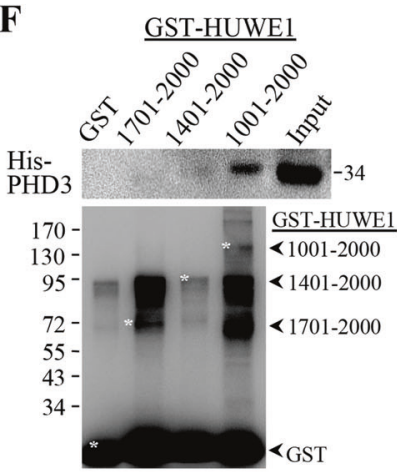

H

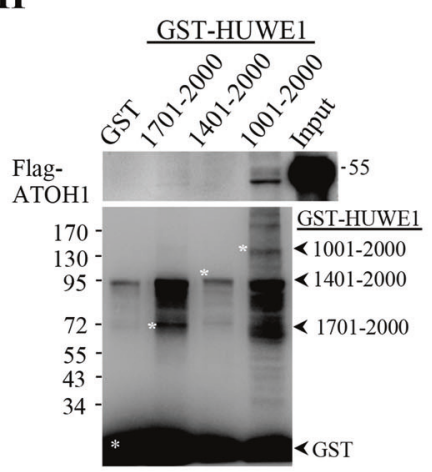

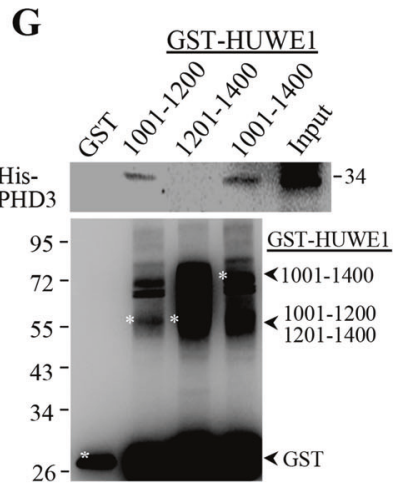

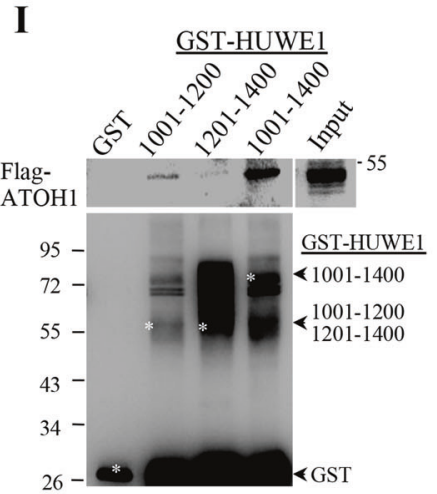

"Methods". The supernatants of $E$. coli containing GST-HUWE1 (1001-2000) were incubated with glutathione-Sepharose beads at $4{ }^{\circ} \mathrm{C}$ for $1 \mathrm{~h}$. Then the beads were washed and incubated at $4{ }^{\circ} \mathrm{C}$ with $E$. coli supernatants containing His-PHD3. After $2 \mathrm{~h}$, the beads were washed and subjected to immunoblotting. f His-PHD3 did not bound GSTHUWE1(1701-2000) and GST-HUWE1(1401-2000). g His-PHD3 interacted with GST-HUWE1(1001-1200). $\mathbf{h}$ The beads that already captured GST-HUWE1 variants were incubated at $4{ }^{\circ} \mathrm{C}$ with $293 \mathrm{~T}$ lysates containing Flag-ATOH1 for $2 \mathrm{~h}$. i Flag-ATOH1 interacted with GST-HUWE1(1001-1200) and GST-HUWE1(1001-1400).

transfected as described above, and the transfected cells were incubated with the protein synthesis inhibitor CHX for different time intervals, followed by determination of Flag-ATOH1 by western blot. The results show that overexpression of PHD3 slowed down the decrease of FlagATOH1 protein in the presence of CHX (Fig. 2i, j). These results suggest that PHD3 stabilizes ATOH1.

\section{PHD3 associated with HUWE1}

We next determined the molecular mechanism underlying ATOH1 stabilization by PHD3. We found that overexpression of PHD3 reduced the ubiquitination of ATOH1 (Fig. 3a). It is known that the E3 ubiquitin-protein ligase HUWE1 mediates ubiquitination and degradation of ATOH1 $[29,30]$. We therefore presumed that PHD3 stabilized ATOH1 through HUWE1. We firstly determined whether 
PHD3 influenced the expression of HUWE1 and found that overexpression of PHD3 had no effect on protein levels of HUWE1 in CCD841 and 293T cells (Fig. 3b). Then, we determined whether PHD3 associated with HUWE1 by performing co-immunoprecipitation experiment. The results show that PHD3 was precipitated by the HUWE1-specific antibody, but not by IgG (Fig. 3c), implying that PHD3 interacts with HUWE1.

The human HUWE1 protein is consisted of 4374 amino acids and has a molecule weight around $481.9 \mathrm{kDa}$. It contains a ubiquitin-associated domain (UBA) (amino acid 1318-1354), a WWE (amino acid 1612-1692), and a Cterminal HECT domain [31]. To know the details of the interaction between PHD3 and HUWE1, we constructed vectors that encoded four truncated GST-HUWE1, GSTHUWE1(1-1000), GST-HUWE1(1001-2000), GSTHUWE1(2001-3000), and GST-HUWE1(3001-4374) (Fig. 3d). Production of these truncated GST-HUWE1 and His-PHD3 in E. coli was as described in "Methods". We found that His-PHD3 associated with GST-HUWE1(10012000) but not other three truncated GST-HUWE1 (Fig. 3e). The HUWE1(1001-2000) fragment contains UBA and WWE domain [31]. We divided this fragment into two smaller parts, GST-HUWE1(1401-2000) and GST-HUWE1 (1701-2000). His-PHD3 did not bind either GST-HUWE1 (1701-2000) or GST-HUWE1(1401-2000) (Fig. 3f), indicating that PHD3 binds HUWE1 at the region of amino acid 1001-1400. Next, we constructed GST-HUWE1(10011400), (1201-1400) and (1201-1400). As expected, PHD3 bound GST-HUWE1(1001-1400) (Fig. 3g). PHD3 bound GST-HUWE1(1001-1200) but not GST-HUWE1 (1201-1400) (Fig. 3g). HUWE1(1001-1200) does not have either UBA or WWE domain. These results indicate that PHD3 does not bind to either UBA or WW domain.

We also determined whether ATOH1 bound the same site of HUWE1 as PHD3 did. Flag-tagged ATOH1 was examined in our work. As PHD3, Flag-ATOH1 associated with GSTHUWE1(1001-2000), but not (1401-2000) or (1701-2000) (Fig. 3h), implying that Flag-ATOH1 binds GST-HUEW1 (1001-1400). Further analysis shows that Flag-ATOH1 bound GST-HUWE1(1001-1400) and (1001-1200) but not (1201-1400) (Fig. 3i). These results indicate that ATOH1 and PHD3 bind the same region of HUWE1.

\section{PHD3 blocked the interaction between HUWE1 and ATOH1}

To confirm that ATOH1 and PHD3 bind HUWE1(10011200), we constructed a vector encoding myc-HUWE1 and employed purified PHD3-HA-His and ATOH1-Flag-His proteins in our work. The purified proteins were shown in Supplementary Fig. 2. Myc-HUWE1 was expressed in 293 T cells. We found that the purified PHD3 and
ATOH1 still bound HUWE1(1001-1200) (Fig. 4a). These data provide more evidence that PHD3 and ATOH1 bind the same site of HUWE1. Based on the results that PHD3 and ATOH1 bound the same site of HUWE1, we proposed that PHD3 might bind HUWE1 competitively and block the interaction of HUWE1-ATOH1. To verify this, we transfected 293T cells with Flag-ATOH1 or Flag-ATOH1 plus Myc-PHD3 vectors and found that overexpression of MycPHD3 suppressed the interaction between HUWE1 and Flag-ATOH1 (Fig. 4b). Myc-PHD3 inhibited the interaction between HUWE1 and Flag-ATOH1 in a dose-dependent manner (Fig. 4c). Overexpression of Myc-PHD3 also impeded the interaction of GST-HUWE1(1001-1200) and Flag-ATOH1 (Fig. 4d). We also employed purified PHD3 and ATOH1 proteins in our work. We found that the interaction between GST-HUWE1(1001-1200) and ATOH1-Flag-His was inhibited by purified PHD3-HA-His (Fig. 4e). Last, we did an in vitro ubiquitination assay to test the competition. Our results show that the purified PHD3HA-His prevented HUWE1 from ubiquitinating purified ATOH1-Flag-His (Fig. 4f). Together, these results suggest that PHD3 prevents HUWE1 from binding ATOH1.

\section{PHD3 stabilized ATOH1 in a hydroxylase- independent manner}

As PHDs have hydroxylase-dependent and -independent functions [5-14], we wanted to know whether the prolyl hydroxylase activity was required for PHD3 to stabilize ATOH1. The histidine residue H196 of PHD3 is critical for its enzymatic activity, and mutation of $\mathrm{H} 196$ to alanine leads to loss of PHD3 prolyl hydroxylase activity [32, 33]. We determined the effect of prolyl hydroxylase-deficient PHD3(H196A) variant on ATOH1 expression. Overexpression of PHD3(H196A) increased the protein level of ATOH1 as the wild type PHD3 did (Fig. 5a). Overexpression of PHD3(H196A) reduced the ubiquitination of Flag-ATOH1 (Fig. 5b). These results suggest that the PHD3 prolyl hydroxylase activity is dispensable for enhancing ATOH1 expression.

\section{PHD(H196A) knock-in did not influence goblet cell number in mice intestine}

To know whether the prolyl hydroxylase activity is required for PHD3 to modulate the formation of goblet cells, we generated the intestinal epithelium Phd3(H196A)-knock-in $\left(P h d 3(\mathrm{H} 196 \mathrm{~A})^{\mathrm{IEC}-\mathrm{KI}}\right)$ mice as described [34]. The mouse PHD3 is similar to human PHD3 (97\% identity) and the mouse PHD3(H196A) also loses prolyl hydroxylase activity [34]. Mice at age of 5 weeks were examined. PHD3(H196A)knockin had little influence on protein levels of ATOH1 and Muc2 (Fig. 5c). PAS stain shows that the amount of PAS- 
A

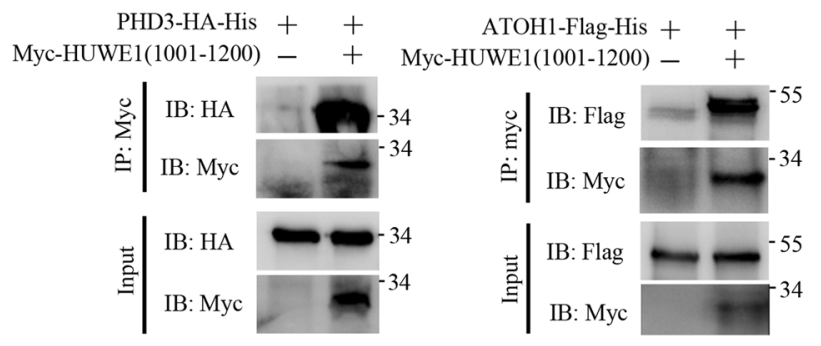

B

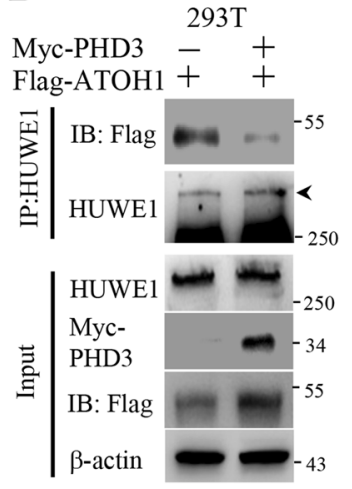

C

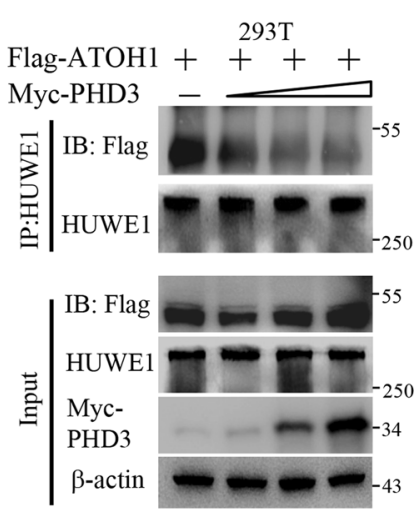

D

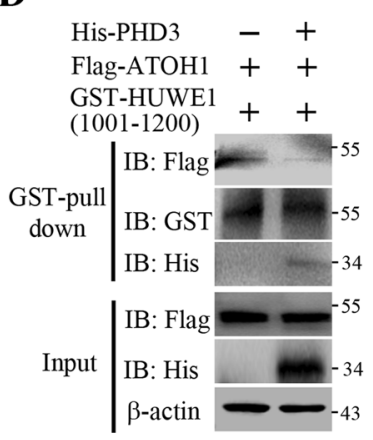

$\mathbf{E}$

F

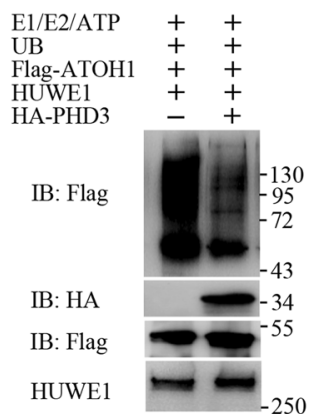

Fig. 4 PHD3 blocked the HUWE1-ATOH1 interaction. a 293 T cells were transfected with myc-HUWE1(1001-1200) vector. After $24 \mathrm{~h}$, cell lysates $(200 \mu \mathrm{g})$ were prepared and incubated with $1 \mu \mathrm{g}$ Myc antibody at $4{ }^{\circ} \mathrm{C}$ for $3 \mathrm{~h}$, followed by incubation with $40 \mu \mathrm{l}$ glutathione-Sepharose beads for another $1 \mathrm{~h}$. The beads were washed and $30 \mu \mathrm{g}$ purified PHD3-HA-His or ATOH1-Flag-His proteins were added and incubated at $4{ }^{\circ} \mathrm{C}$ overnight. The beads were washed, boiled in SDS-PAGE loading buffer, and the resolved proteins were subjected to western blot. b $293 \mathrm{~T}$ cells were transfected with Myc-PHD3 or Myc-PHD3 plus Flag-ATOH1 vector. Twenty-four hours post transfection, the cells were treated with MG132 $(10 \mu \mathrm{M})$ for $4 \mathrm{~h}$, followed by immunoprecipitation experiment. c $293 \mathrm{~T}$ cells were transfected with Flag-ATOH1 and different amount of Myc-PHD3 (0, 0.5, 1 or

positive cells in $P$ hd3(H196A) ${ }^{\mathrm{IEC}-\mathrm{KI}}$ mice intestine was similar to that in control littermates (Fig. 5d). We immunostained mice colon tissues with Muc2 antibody, and found that PHD3 (H196A) knock-in had little effect on the amount of Muc2positive cells (Fig. 5e). These results suggest that PHD3 modulates goblet cell generation independent of its hydroxylase activity. A working model for PHD3 is proposed (Fig. 5f). We also determined the effect of PHD3(H196A) knock-in on formation of Paneth and enteroendocrine cells in mice small intestines. The results indicate that the formation of these cells was not influenced (Supplementary Fig. 3).

\section{PHD3(H196A) knock-in had little effect on DSS-induced colitis in mice}

Our previous work shows that knockout of PHD3 in intestinal epithelial cells sensitized mice to dextran sulfate

$2 \mu \mathrm{g})$ vector. After $24 \mathrm{~h}$, the cells were treated with MG132 $(10 \mu \mathrm{M})$ for $4 \mathrm{~h}$, followed by immunoprecipitation assay. d The beads that captured GST-HUWE1(1001-1200) were incubated at $4{ }^{\circ} \mathrm{C}$ with $293 \mathrm{~T}$ lysates containing Flag-ATOH1 for $2 \mathrm{~h}$. The beads were washed and incubated with bacteria lysates containing His-PHD3 at $4{ }^{\circ} \mathrm{C}$ overnight. e GST-HUWE1(1001-1200) was incubated with glutathioneSepharose beads at $4{ }^{\circ} \mathrm{C}$ for $1 \mathrm{~h}$. The beads were washed and incubated with ATOH1-Flag-His $(18 \mu \mathrm{g})$ or equal amount of ATOH1-Flag-His plus PHD3-HA-His at $4{ }^{\circ} \mathrm{C}$ overnight. The beads were washed, boiled in SDS-PAGE loading buffer, and the resolved proteins were detected by immunoblotting. f Purified PHD3-HA-His inhibited the ubiquitination of ATOH1-Flag-His by HUWE1 in vitro. The experiment was performed as described in "Methods".

sodium (DSS) colitis [26]. We wanted to know whether a genetic knockin of PHD3(H196A) had any effect on mice DSS colitis. We treated the $P h d 3^{\mathrm{IEC}-\mathrm{KO}}$ mice, $P h d 3(\mathrm{H} 196 \mathrm{~A})$ IEC-KI mice, and their control littermates with DSS as described in "Methods". In line with our previous results, the Phd $3^{\mathrm{IEC}-\mathrm{KO}}$ mice were more sensitive to DSS treatment than control littermates, as evidenced by reduced body weight, decreased colon length, and increased disease activity index (Fig. 6a-d). Histological analysis shows that the colon epithelium of $P h d 3^{\mathrm{IEC}-\mathrm{KO}}$ mice had more crypt loss, lamina propria collapse, areas of mucosal erosion, and lymphocyte infiltration than control littermates did (Fig. 6e). DSS treatment increased significantly the expression levels

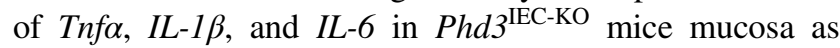
compared with control littermates (Fig. 6f). While, when subjected to DSS treatment, the Phd $3(\mathrm{H} 196 \mathrm{~A})^{\mathrm{IEC}-\mathrm{KI}}$ mice exhibited similar wasting, colon shortening, and disease 
A

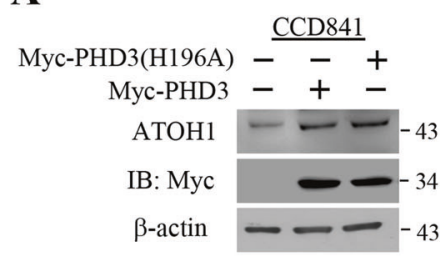

B

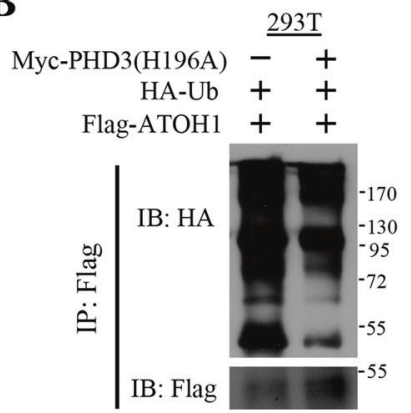

C

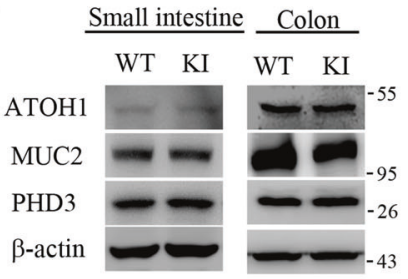

D
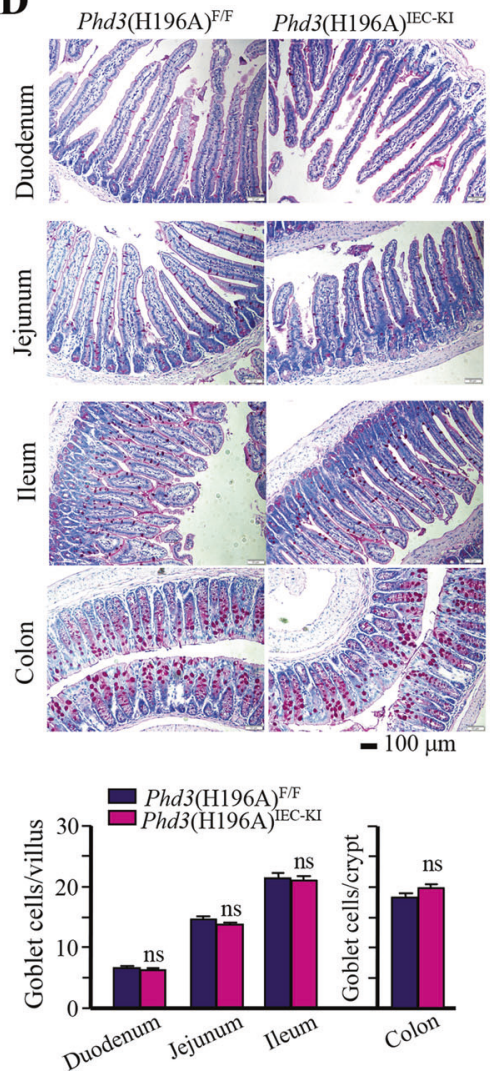

$\mathbf{E}$

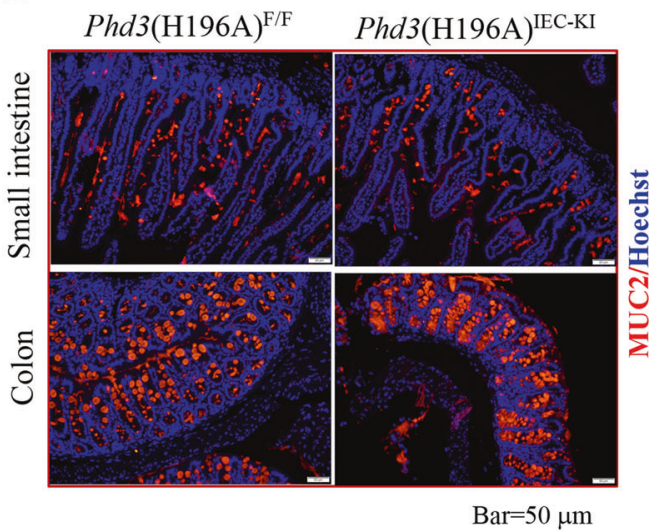

$\mathbf{F}$

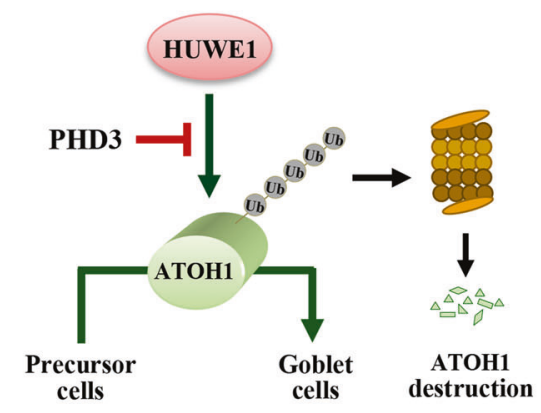

Fig. 5 PHD3 controlled goblet cell generation independent of its hydroxylase activity. a CCD841 cells were transfected with MycPHD3 or Myc-PHD3(H196A). After 24 h, the cells were harvested for determination of ATOH1. b Overexpression of PHD3(H196A) reduced ubiquitination of ATOH1. 293T cells were transfected with Myc- PHD3(H196A) or Myc-PHD3(H196A) plus Flag-ATOH1 vectors in the presence of HA-Ub vector. After $24 \mathrm{~h}$, the cells were treated with MG132 $(10 \mu \mathrm{M})$ for $4 \mathrm{~h}$, followed by immunoprecipitation. c Determination of the expression of ATOH1 and Muc2 in mice small

activity index as the control littermates did (Fig. 6a-d). Histological analysis shows that the colon epithelium of Phd3(H196A) $)^{\mathrm{IEC}-\mathrm{KI}}$ mice had same crypt loss, lamina propria collapse, areas of mucosal erosion, and lymphocyte infiltration, as compared with the control littermates (Fig. 6e). The expression levels of Tnf $\alpha, \mathrm{Il}-1 \beta$, and Il-6 in colon mucosa of the PHD3(H196A)-knock-in mice are the same as those from control littermates (Fig. 6f). These data indicate that the genetic knockin of PHD3(H196A) had no effect on DSS-induced colitis in mice.

\section{Discussion}

In this paper, we delineate a role of PHD3 in the regulation of intestinal goblet cells generation. We used a combination of genetic and cellular techniques to characterize the function of PHD3. We found that genetic ablation of PHD3 reduced the amount of intestine goblet cells in mice, but a intestine and colon epithelial cells. d PAS stain of intestinal epithelia from Phd3(H196A) $)^{\mathrm{IEC}-\mathrm{KI}}$ mice and control littermates. Male mice at age of 5 weeks old (the body weight of the mice is within normal limits) were selected randomly $(n=3)$. The down panel shows the number of goblet cells from mice intestinal epithelia. e Stain of mice intestinal epithelia with Muc2 antibody. f A proposed model of PHD3 regulating the ATOH1 expression and goblet cell generation. ns no significance.

genetic knockin of PHD3(H196A) had little effect. Mechanistically, PHD3 inhibited HUWE1-ATOH interaction and prevented HUWE1 from mediating ATOH1 degradation, leading to ATOH1 stabilization. Our results suggest that PHD3 plays a role in controlling intestinal goblet cell generation through stabilizing ATOH1 independent of its prolyl hydroxylase activity.

Immature intestinal epithelial cells that are produced by the stem cells differentiate into absorptive or secretory cells. Notch signaling plays a central role in differentiation of these precursor cells into the secretory lineage in intestine $[16,17]$. Activation of Notch signaling suppresses goblet cell fate through activating the expression of HES1 [19]. While the transcription factor ATOH1 plays a reciprocal role to promote goblet cell differentiation [18]. ATOH1 is transcriptionally repressed by HES1 [23]. Our results indicate that PHD3 did not modulate goblet cell generation through Notch signaling, as PHD3 deficiency had little effect on the expression of NICD and HES1 (Fig. 2). 
A

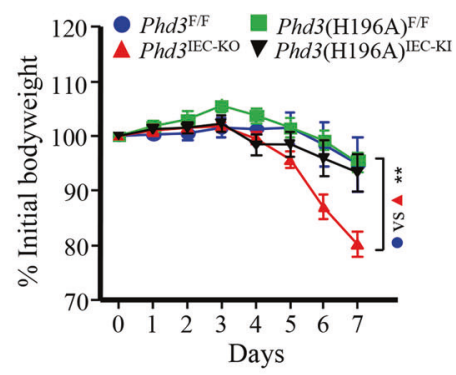

D

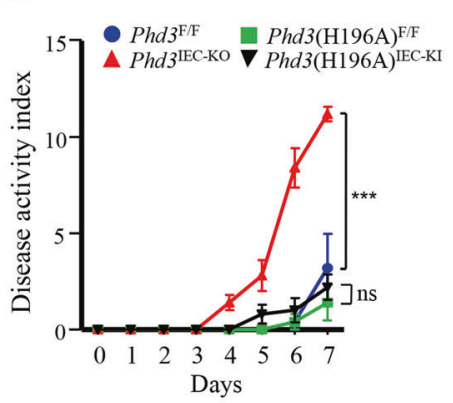

B

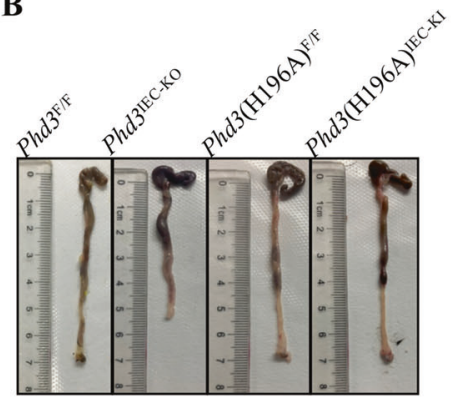

$\mathbf{E}$

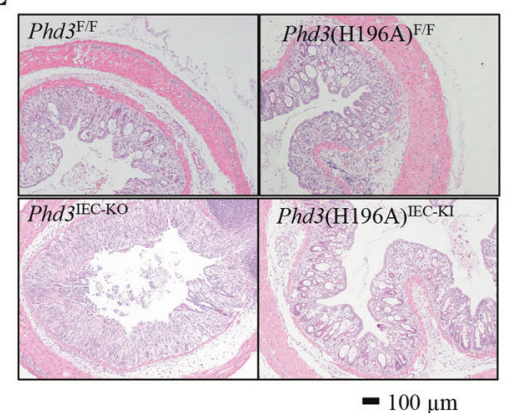

C

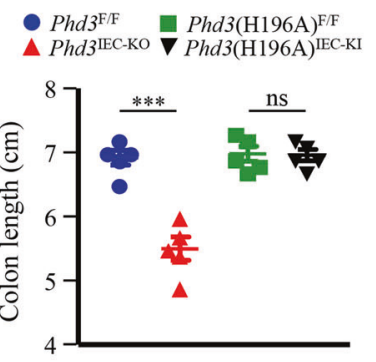

F

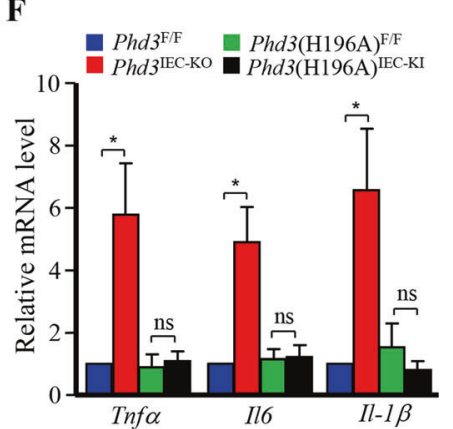

Fig. 6 PHD(H196A) knock-in had little effect on DSS-induced colitis. a-f Age-matched (8-10 weeks old) male littermates (the body weight of the mice is within normal limits) were treated with DSS ( $2.5 \%$ in drinking water) for 7 days. After treatment, the mice were sacrificed. Each group had five mice. a Mice body weight. Data are mean \pm SEM. The $P$ value was calculated using two-way ANOVA. b Representative of mice colons. $\mathbf{c}$ The colon length of mice. Data are

Interestingly, we found that loss of PHD3 decreased ATOH1 protein without influencing its transcription, implying that PHD3 regulates the expression of ATOH1 in a way rather than Notch signaling. We found that PHD3 bound the same site of HUWE1 as ATOH1 did. It impeded the HUWE1-ATHO1 interaction, thus preventing HUWE1 from mediating ATHO1 ubiquitination and destruction (Figs. 2-4). These data reveal an additional critical mechanism underlying the regulation of ATOH1 expression and thereafter goblet cell generation by PHD3.

Goblet cells have been implicated as a player in promoting intestinal homeostasis and host defense [35, 36]. They play an important role in host protection through secreting Muc2 and other factors. Muc2 is prominent in the gut and it forms a mucus gel layer to protect the intestinal epithelium against physical and chemical injury. Muc2 deficiency led to spontaneous colitis in mice [37]. It was found that expression of Muc2 was decreased in human inflammatory bowel diseases [38] and Muc2 expression is inversely correlated with the severity of inflammation [39]. We found that loss of PHD3 decreased goblet cell number and inhibited Muc2 production (Fig. 1). These data may explain our previous finding that PHD3 deficiency impaired the intestinal barrier function [26]. ATOH1 is also critical for generation of other intestinal secretory cells including mean \pm SEM. The $P$ value was calculated using two-way ANOVA. d Disease activity index of mice. Data are mean \pm SEM. Statistical analysis was performed using two-way ANOVA. e H\&E staining of colon tissue sections of mice. $\mathbf{f}$ Determination of mRNA levels of Tnf $\alpha, I l-1 \beta$, and $I l-6$ in mice colon mucosa $(n=5)$. The data are mean \pm SEM. Scale bar $=100 \mu \mathrm{m}$. $* * P<0.01 ; * * * P<0.001$. ns no significance.

Paneth and enteroendocrine cells [22, 27, 28]. So, PHD3 may also play a role in regulating the generation of Paneth and enteroendocrine cells. We do found in our work that PHD3-knockout reduced the formation of these two cells (Supplementary Fig. 1).

PHDs are initially recognized as prolyl hydroxylases that catalyze hydroxylation of proteins including HIF- $1 \alpha$. Recent studies indicate that PHDs also have prolyl hydroxylase-independent functions. Chan et al. [40] demonstrated that PHD2 could regulate angiogenesis in a hydroxylase-independent manner. Fu et al. [11] reported that PHD3 competed with cIAP1 for IKK $\gamma$ binding, leading to inhibition of cIAP1-IKK $\gamma$ interaction, IKK $\gamma$ ubiquitination, and IKK/NF- $\mathrm{KB}$ signaling independent of its hydroxylase activity. We found recently that PHD3 regulated expression of the tight junction protein occludin in a hydroxylase-independent mechanism [26]. These findings drove us to investigate whether the prolyl hydroxylase activity was required for PHD3 to regulate ATOH1 expression and goblet cell generation. Overexpression of the prolyl hydroxylase activity-deficient variant PHD3 (H196A) induced the expression of ATHO1 (Fig. 5a) and inhibited ATOH1 ubiquitination (Fig. 5b). These results indicate that the prolyl hydroxylase activity of PHD3 is dispensable for its ability to modulate ATOH1 expression. 
As ATOH1 is an essential factor controlling goblet cell differentiation, we presumed that PHD3 might modulate goblet cell generation independent of its prolyl hydroxylase activity. We employed $P h d 3^{\mathrm{IEC}-\mathrm{KO}}$ and $P h d 3(\mathrm{H} 196 \mathrm{~A})^{\mathrm{IEC}-\mathrm{KI}}$ mice in our work. Genetic ablation of PHD3 in mice intestinal epithelial cells reduced the amount of intestine goblet cells, but a genetic knockin of PHD3(H196) had little effect (Fig. 5c-e). These data imply that PHD3 controls goblet cell generation independent of its hydroxylase activity. Moreover, we found that knockout of PHD3 sensitized mice to DSS colitis, while a genetic knockin of PHD3(H196A) did not (Fig. 6). These results suggest that the prolyl hydroxylase activity of PHD3 is dispensable for its ability to maintain intestinal epithelial homeostasis.

In this paper, we reveal an additional critical mechanism underling the regulation of ATOH1 expression by PHD3, and highlight that PHD3 plays a role in controlling the generation of intestine goblet cells.

\section{Materials and methods}

\section{Animals}

C57BL/6J mice were purchased from Shanghai Experimental Animal Center. The intestinal epithelia-specific Phd3 knockout mice $\left(P h d 3^{\text {flox/llox }}:\right.$ Villin-Cre, $\left.P h d 3^{\mathrm{IEC}-\mathrm{KO}}\right)$ were generated by intercrossing the $P h d 3^{\text {flox/lox }}\left(P h d 3^{\mathrm{F} / \mathrm{F}}\right)$ mice with VillinCre ones as described [26]. The Phd3(H196A) $)^{\mathrm{IEC}-\mathrm{KI}}$ mice were generated by intercrossing the $P h d 3(\mathrm{H} 196 \mathrm{~A})^{\text {flox/flox }}$ $\left(P h d 3(\mathrm{H} 196 \mathrm{~A})^{\mathrm{F} / \mathrm{F}}\right)$ mice with Villin-Cre mice. The Phd3 $(\mathrm{H} 196 \mathrm{~A})^{\mathrm{F} / \mathrm{F}}$ mice were created at Shanghai Research Center for Model Organisms. The details for generation of the mice were shown [34]. All animals were housed and maintained in laboratory cages in a 12-h dark/light cycle with a free access to a regular chow diet under a specified pathogen-free condition. All experimental protocols were approved by the Institutional Animal Care and Use Committee at the Institute for Nutritional Sciences, Shanghai Institutes for Biological Sciences, Chinese Academy of Sciences.

\section{Cell culture}

Human normal colon epithelial CCD841 cells were grown in RPMI-1640 medium. All medium were supplemented with $10 \%$ fetal bovine serum, $100 \mu / \mathrm{ml}$ penicillin, and 100 $\mu \mathrm{g} / \mathrm{ml}$ streptomycin. The cells were cultured at $37{ }^{\circ} \mathrm{C}$ in an incubator $\left(5 \% \mathrm{CO}_{2}\right)$.

\section{Reagents and antibodies}

PHD3(NB100-303) and HUWE1(NB100-652) antibodies were products of Novus Biologicals (Littleton, $\mathrm{CO}$ ). Muc2
(H300), His, HA and Myc antibodies were from Santa Cruz Biotechnology (Santa Cruz, CA). Muc2(ab134119), NICD (ab8925), HES1(ab71559), and ATOH1(ab137534) antibodies were from Abcam. Flag (M20008) antibody was from Abmart. $\beta$-actin antibody was from Sigma (St. Louis, MO). Cycloheximide (CHX) and DSS were purchase form Sigma and MP Biomedicals, respectively. DMOG was from Selleck.

\section{Construction of vectors}

The vectors encoding PHD3 and hydroxylase-deficient PHD3(H196A) were constructed as described previously [8]. The vectors encoding glutathione S-transferase (GST)HUWE1 fusion proteins were constructed by inserting PCR-generated DNA fragments encoding regions of HUWE1 into pGEX-4T-1. The vector encoding Flag-tagged ATOH1 was constructed by inserting coding sequence of ATOH1 into pCMVtag2B. Construction of vectors encoding mice PHD3 and PHD3(H196A) was as described [34].

\section{Small-interfering RNA}

Small-interfering RNA (siRNA) duplexes were synthesized by Gene Pharma (Shanghai, China). The cells were transfected with siRNA oligos by using LipofectAMINE-2000. The sequences of siRNA oligos are as described [8].

\section{Quantitative real-time PCR (qPCR)}

qPCR was performed as described [8]. Beta-actin was used as the internal control. The primers for mouse genes are as follows:

Atoh1: 5'CAGGGTGAGCTGGTAAGGAG3'(F), 5'GC CAAGCTCGTCCACTACA3'(R); Phd3: 5'GATGGCCGC TGTATCACCTG3'(F), 5'AGGGCTGGACTTCATGTGG A3'(R); Tnfo: 5'CCCTCACACTCAGATCATCTTCT3' (F), 5'GCTACGACGTGGGCTACAG3'(R); Il-6: 5'TAG TCCTTCCTACCCCAATTTCC3'(F), 5'TTGGTCCTTAG CCACTCCTTC3'(R); IL-1 $\beta$ : 5GCAACTGTTCCTGAA CTCAACT3(F), 5ATCTTTTGGGGTCCGTCAACT3'(R); $\beta$-actin: 5'GATCATTGCTCCTCCTGAGC3'(F), 5'ACTC CTGCTTGCTGATCCAC3'(R). The primers for human genes are ATOH1: 5'CCAGCTGCGCAATGTTATCC3' (F), 5'TCGGACAAGGCGTTGATGTA3'(R); PHD3: 5'A TCAGCTTCCTCCTGTCCC3'(F), 5'CAGCGACCATCA CCGTTG3'(R); $\beta$-actin: 5'GATCATTGCTCCTCCTGAG C3'(F), 5'ACTCCTGCTTGCTGATCCAC3'(R).

\section{GST-pull-down assay}

Bacterial cells were lysed by using the following buffer: $20 \mathrm{mmol} / \mathrm{l}$ Tris-Cl, $150 \mathrm{mmol} / \mathrm{l} \mathrm{NaCl}, 2 \mathrm{mmol} / \mathrm{l}$ EDTA, 
and $0.5 \%$ NP40, $\mathrm{pH}$ 7.5. To determine the interaction between HUWE1 and target proteins, bacterial lysates containing GST-HUWE1 were incubated with glutathione-Sepharose $4 \mathrm{~B}$ beads at $4{ }^{\circ} \mathrm{C}$ for $1 \mathrm{~h}$. The beads were washed and incubated with target proteins, allowing the interaction between GST-HUWE1 and target proteins. After washing, GST-HUWE1 and the bound target proteins were eluted from the beads and subjected to electrophoresis.

\section{Protein stability assay}

Determination of protein stability was performed as described [26]. In brief, 293T cells were transfected with Flag-ATOH1 + empty vector or Flag-ATOH1 + MycPHD3. After $24 \mathrm{~h}$, CHX $(10 \mu \mathrm{g} / \mathrm{ml})$ was added to block protein synthesis. Cells were harvested at different time points. Equal amounts of protein from each treatment were used for immunoblotting. Protein bands were quantified by densitometry. The relative protein level of Flag-ATOH1 was determined and normalized to that of actin. The relative Flag-ATOH1 protein level at time zero is designated as 1 .

\section{Preparation of purified PHD3-HA-His and ATOH1-Flag-His proteins}

Purified PHD3-HA-His and ATOH1-Flag-His proteins were prepared by NovoPro Bioscience (Shanghai, CHINA). In brief, PHD3-HA-His and ATOH1-Flag-His were expressed in Escherichia coli Rosetta (DE3). The pET22b vector was used to express these proteins. Expression of PHD3-HA-His and ATOH1-Flag-His proteins was induced by $1 \mathrm{mM}$ IPTG at $25^{\circ} \mathrm{C}$ for $12 \mathrm{~h}$. These proteins were purified in a Ni-IDASepharose CL-6B column.

\section{In vitro ATOH1 ubiquitination assay}

The in vitro ATOH1 ubiquitination assay was performed as described [34]. E1(UBE1)(Catalog No. E-305), E2 (UBE2D3)(Catalog No. E2-627), ATP-Mg ${ }^{2+}$ (Catalog No. SK-10), and Ub (Catalog No. U-100H) were purchased from BostonBiochem. 293T cell lysates $(400 \mu \mathrm{g})$ were incubated with $1 \mu \mathrm{g}$ of HUWE1 antibody at $4{ }^{\circ} \mathrm{C}$ for $3 \mathrm{~h}$. Eighty microliters of protein A/G Plus Agarose beads were added and the incubation continued at $4{ }^{\circ} \mathrm{C}$ overnight. The beads were washed and split equally into two tubes in the presence or absence of $7.5 \mu \mathrm{g}$ purified His-tagged PHD3-HA (in $600 \mu \mathrm{l}$ buffer). The beads were incubated at $4{ }^{\circ} \mathrm{C}$ for $1 \mathrm{~h}$. The beads were washed and resuspended in a reaction volume of $30 \mu \mathrm{l}$ containing $3 \mu \mathrm{l}$ of $10 \times$ reaction buffer, $10 \mathrm{ng}$ of E1, $10 \mathrm{ng}$ of E2, $3 \mu \mathrm{l}$ of $10 \times \mathrm{Mg}^{2+}$-ATP solution, $3 \mu \mathrm{l}$ of $10 \times \mathrm{Ub}$, and $2.5 \mu \mathrm{g}$ of purified His-tagged
ATOH1-Flag. The mixture was incubated at $37^{\circ} \mathrm{C}$ for $1 \mathrm{~h}$. After the reaction completed, the supernatant was used for ATOH1 ubiquitination analysis in a western blot. The beads were boiled in loading buffer, resolved in SDS-PAGE, and immunoblotted with HA and HUWE1 antibody.

\section{Isolation of intestinal epithelial cells}

The intestine was removed and washed free of fecal material with solution " $\mathrm{A}$ " $(96 \mathrm{mM} \mathrm{NaCl}, 27 \mathrm{mM}$ sodium citrate, $1.5 \mathrm{mM} \mathrm{KCl}, 0.8 \mathrm{mM} \mathrm{KH} \mathrm{PO}_{4}, 5.6 \mathrm{mM} \mathrm{Na}_{2} \mathrm{HPO}_{4}, 5000 \mathrm{U} / \mathrm{l}$ penicillin, $5 \mathrm{mg} / \mathrm{l}$ streptomycin, $0.5 \mathrm{mM}$ DTT, and $2 \mathrm{mM}$ phenylmethylsulfonyl fluoride, $\mathrm{pH}$ 7.4). Square pieces of tissue were placed in solution $\mathrm{A}(10 \mathrm{ml})$ at $37^{\circ} \mathrm{C}$ for $10 \mathrm{~min}$ with gentle shaking. This removed the mucus, bacteria, and other lumen contents. The tissue fragments were then incubated in solution " $\mathrm{B}$ " $(0.1 \mathrm{mM}$ EDTA, $115 \mathrm{mM} \mathrm{NaCl}$,

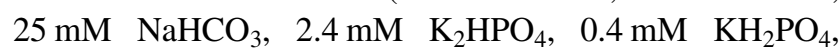
$0.5 \mathrm{mM}$ DTT, $5 \mathrm{mg} / 1$ streptomycin, $2.5 \mathrm{mM}$ glutamine, $5000 \mathrm{U} / 1$ penicillin, and $2 \mathrm{mM}$ phenylmethylsulfonyl fluoride, $\mathrm{pH} \mathrm{7.4)}$ at $37^{\circ} \mathrm{C}$ for $30 \mathrm{~min}$; the disruption of the mucosa and elution of cells was stopped by adjusting to $1 \mathrm{mM} \mathrm{CaCl}{ }_{2}$. The cells recovered in the suspension were collected by centrifugation and lysed in RIPA buffer.

\section{Tissue staining}

Colon tissues were formalin-fixed and paraffin-embedded, and hematoxylin eosin (HE) staining was performed. The PAS staining was performed for detection of goblet cells as described [41]. Immunohistochemical staining was performed as described [8].

\section{DSS colitis}

Mice DSS colitis was performed as described previously [26]. In brief, the sex- and age-matched male littermates (8-10 weeks old) received DSS (2.5\%) in drinking water for 7 days. The mice body weight was recorded daily. The disease activity index was determined as described [25].

\section{Statistical analysis}

Statistical analysis was made by using the unpaired two-tailed Student's $t$ test or two-way analysis of variance (ANOVA) with GraphPad Prism 5.0. The data represent mean \pm SEM from three independent experiments except where indicated. $P<0.05$ is considered statistically significant.

Acknowledgements This work was supported by China Ministry of Science and Technology (2017YFD0400206), National Natural Science Foundation of China $(31470769,31670785)$, and Shanghai Ministry of Science and Technology (16JC1406100). 


\section{Compliance with ethical standards}

Conflict of interest The authors declare that they have no conflict of interest.

Publisher's note Springer Nature remains neutral with regard to jurisdictional claims in published maps and institutional affiliations.

\section{References}

1. Schofield CJ, Ratcliffe PJ. Oxygen sensing by HIF hydroxylases. Nat Rev Mol Cell Biol. 2004;5:343-54.

2. Kaelin WG Jr, Ratcliffe PJ. Oxygen sensing by metazoans: the central role of the HIF hydroxylase pathway. Mol Cell. 2008;30:393-402.

3. Fong GH, Takeda K. Role and regulation of prolyl hydroxylase domain proteins. Cell Death Differ. 2008;15:635-41.

4. Metzen E, Berchner-Pfannschmidt U, Stengel P, Marxsen JH, Stolze I, Klinger M, et al. Intracellular localisation of human HIF$1 \alpha$ hydroxylases: implications for oxygen sensing. J Cell Sci. 2003;116:1319-26.

5. Köditz J, Nespe J, Wottawa M, Stiehl DP, Camenisch G, Franke $\mathrm{C}$, et al. Oxygen-dependent ATF-4 stability is mediated by the PHD3 oxygen sensor. Blood. 2007;110:3610-7.

6. Fu J, Menzies K, Freeman RS, Taubman MB. EGLN3 prolyl hydroxylase regulates skeletal muscle differentiation and myogenin protein stability. J Biol Chem. 2007;282:12410-8.

7. Xie L, Xiao K, Whalen EJ, Forrester MT, Freeman RS, Fong G, et al. Oxygen-regulated 2-adrenergic receptor hydroxylation by EGLN3 and ubiquitylation by pVHL. Sci Signal. 2009;2:ra33.

8. Xue J, Li X, Jiao S, Wei Y, Wu G, Fang J. Prolyl hydroxylase-3 is down-regulated in colorectal cancer cells and inhibits IKK $\beta$ independent of hydroxylase activity. Gastroenterology. 2010;138:606-15.

9. Luo W, Hu H, Chang R, Zhong J, Knabel M, O’Meally R, et al. Pyruvate kinase M2 is a PHD3-stimulated coactivator for hypoxia-inducible factor 1 . Cell. 2011;145:732-44.

10. Xie L, Pi X, Mishra A, Fong G, Peng J, Patterson C. PHD3dependent hydroxylation of HCLK2 promotes the DNA damage response. J Clin Investig. 2012;122:2827-36.

11. Fu J, Taubman MB. EGLN3 inhibition of NF-kB is mediated by prolyl hydroxylase-independent inhibition of IкB kinase $\gamma$ ubiquitination. Mol Cell Biol. 2013;33:3050-61.

12. Deschoemaeker S, Di Conza G, Lilla S, Martín-Pérez R, Mennerich D, Boon L, et al. PHD1 regulates p53-mediated colorectal cancer chemoresistance. EMBO Mol Med. 2015; 7:1350-65.

13. Rodriguez J, Herrero A, Li S, Rauch N, Quintanilla A, Wynne K, et al. PHD3 regulates p53 protein stability by hydroxylating proline 359. Cell Rep. 2018;24:1316-29.

14. Hiwatashi Y, Kanno K, Takasaki C, Goryo K, Sato T, Torii S, et al. PHD1 interacts with ATF4 and negatively regulates its transcriptional activity without prolyl hydroxylation. Exp Cell Res. 2011;317:2789-99.

15. Maloy KJ, Powrie F. Intestinal homeostasis and its breakdown in inflammatory bowel disease. Nature. 2011;474:298-306.

16. Fre S, Huyghe M, Mourikis P, Robine S, Louvard D, ArtavanisTsakonas S. Notch signals control the fate of immature progenitor cells in the intestine. Nature. 2005;435:964-8.

17. Sancho R, Cremona CA, Behrens A. Stem cell and progenitor fate in the mammalian intestine: Notch and lateral inhibition in homeostasis and disease. EMBO Rep. 2015;16:571-81.
18. Yang Q, Bermingham NA, Finegold MJ, Zoghbi HY. Requirement of Math1 for secretory cell lineage commitment in the mouse intestine. Science. 2001;294:2155-8.

19. Pellegrinet L, Rodilla V, Liu Z, Chen S, Koch U, Espinosa L, et al. Dll1-and dll4-mediated notch signaling are required for homeostasis of intestinal stem cells. Gastroenterology. 2011; 140:1230-40.

20. Jensen J, Pedersen EE, Galante P, Hald J, Heller RS, Ishibashi M, et al. Control of endodermal endocrine development by Hes- 1 . Nat Genet. 2000;24:36-44.

21. Shields JM, Christy RJ, Yang VW. Identification and characterization of a gene encoding a gut-enriched Kruppel-like factor expressed during growth arrest. J Biol Chem. 1996; 271:20009-17.

22. Vandussen KL, Samuelson LC. Mouse atonal homolog 1 directs intestinal progenitors to secretory cell rather than absorptive cell fate. Dev Biol. 2010;346:215-23.

23. Elise S, Demitrack ES, Linda C, Samuelson LC. Notch regulation of gastrointestinal stem cells. J Physiol. 2016;594:4791-803.

24. Robinson A, Keely S, Karhausen J, Gerich ME, Furuta GT, Colgan SP. Mucosal protection by hypoxia-inducible factor prolyl hydroxylase inhibition. Gastroenterology. 2008; 134:145-55.

25. Tambuwala MM, Cummins EP, Lenihan CR, Kiss J, Stauch M, Scholz CC, et al. Loss of prolyl hydroxylase-1 protects against colitis through reduced epithelial cell apoptosis and increased barrier function. Gastroenterology. 2010;139:2093-101.

26. Chen Y, Zhang HS, Fong GH, Xi QL, Wu GH, Bai CG, et al. PHD3 stabilizes the tight junction protein occludin and protects intestinal epithelial barrier function. J Biol Chem. 2015; 290:20580-9.

27. Hollingsworth MA, Swanson BJ. Mucins in cancer: protection and control of the cell surface. Nat Rev Cancer. 2004; $4: 45-60$.

28. Shroyer NF, Helmrath MA, Wang VY, Antalffy B, Henning SJ, Zoghbi HY. Intestine-specific ablation of mouse atonal homolog 1 (ATOH1) reveals a role in cellular homeostasis. Gastroenterology. 2007;132:2478-88.

29. Forget A, Bihannic L, Cigna SM, Lefevre C, Remke M, Barnat M, et al. Shh signaling protects Atoh1 from degradation mediated by the E3 ubiquitin ligase HUWE1 in neural precursors. Dev Cell. 2014;29:649-61.

30. Cheng YF, Tong M, Edge AS. Destabilization of Atoh1 by E3 ubiquitin ligase Huwe1 and casein kinase 1 Is essential for normal sensory hair cell development. J Biol Chem. 2016; 291:21096-109.

31. Chen D, Kon N, Li M, Zhang W, Qin J, Gu W. ARF-BP1/Mule is a critical mediator of the ARF tumor suppressor. Cell. 2005;121:1071-83.

32. Bruick RK, McKnight SL. A conserved family of prolyl-4hydroxylases that modify HIF. Science. 2001;294:1337-40.

33. Lee S, Nakamura E, Yang H, Wei W, Linggi MS, Sajan MP, et al. Neuronal apoptosis linked to EgIN3 prolyl hydroxylase and familial pheochromocytoma genes:developmental culling and cancer. Cancer Cell. 2005;8:155-67.

34. Xu Y, Gao Q, Xue Y, Li X, Xu L, Li C, et al. Prolyl hydroxylase 3 stabilizes the p53 tumor suppressor by inhibiting the p53MDM2 interaction in a hydroxylase- independent manner. J Biol Chem. 2019;294:9949-58.

35. Birchenough GM, Nyström EE, Johansson ME, Hansson GC. A sentinel goblet cell guards the colonic crypt by triggering Nlrp6dependent Muc2 secretion. Science. 2016;352:1535-42.

36. Allaire JM, Morampudi V, Crowley SM, Stahl M, Yu H, Bhullar $\mathrm{K}$, et al. Frontline defenders: goblet cell mediators dictate 
host-microbe interactions in the intestinal tract during health and disease. Am J Physiol Gastrointest Liver Physiol. 2018;314: G360-G377.

37. Van der Sluis M, De Koning BA, De Bruijn AC, Velcich A, Meijerink JP, Van Goudoever JB, et al. Muc2-deficient mice spontaneously develop colitis, indicating that Muc2 is critical for colonic protection. Gastroenterology. 2006;131:117-29.

38. Tytgat KM, Opdam FJ, Einerhand AW, Buller HA, Dekker J. Muc2 is the prominent colonic mucin expressed in ulcerative colitis. Gut. 1996;38:554-63.
39. Shaoul R, Okada Y, Cutz E, Marcon MA. Colonic expression of Muc2, MUC5AC, and TFF1 in inflammatory bowel disease in children. J Pediatr Gastroenterol Nutr. 2004;38:488-93.

40. Chan DA, Kawahara TL, Sutphin PD, Chang HY, Chi JT, Giaccia AJ. Tumor vasculature is regulated by PHD2-mediated angiogenesis and bone marrow-derived cell recruitment. Cancer Cell. 2009; 15:527-38.

41. van Es JH, de Geest N, van de Born M, Clevers H, Hassan BA. Intestinal stem cells lacking the ATOH1 tumour suppressor are refractory to Notch inhibitors. Nat Commun. 2010;1:18. 